\title{
Diagnostic dose determination and efficacy of chlorfenapyr and clothianidin insecticides against Anopheles malaria vector populations of western Kenya
}

Silas Agumba ${ }^{1,2^{*}}$, John E. Gimnig ${ }^{3}$, Lilian Ogonda ${ }^{1}$, Maurice Ombok ${ }^{2}$, Jackline Kosgei ${ }^{2}$, Stephen Munga ${ }^{2}$, Benard Guyah', Seline Omondi ${ }^{2}$ and Eric Ochomo ${ }^{2}$

\begin{abstract}
Background: Malaria vector control is dependent on chemical insecticides applied to walls by indoor residual spraying or on long-lasting insecticidal nets. The emergence and spread of insecticide resistance in major malaria vectors may compromise malaria control and elimination efforts. The aim of this study was to estimate a diagnostic dose for chlorfenapyr (class: pyrrole) and clothianidin (class: neonicotinoid) and assess the baseline susceptibility of three major Anopheles malaria vectors of western Kenya to these two insecticides.
\end{abstract}

Methods: The Centers for Disease Control and Prevention (CDC) bottle assay was used to determine the diagnostic doses of chlorfenapyr and clothianidin insecticides against the susceptible Kisumu strain of Anopheles gambiae. Probit analysis was used to determine the lethal doses at which 50\% (LD50) and 99\% (LD99) of the susceptible mosquitoes would be killed 24, 48 and $72 \mathrm{~h}$ following exposure for $1 \mathrm{~h}$. Insecticidal efficacy of chlorfenapyr, clothianidin and the pyrethroid deltamethrin was then evaluated against field collected female Anopheles mosquitoes sampled from Nyando, Bumula and Ndhiwa sub-Counties in western Kenya. Members of Anopheles funestus and An. gambiae complexes were identified using polymerase chain reaction (PCR).

Results: The determined diagnostic doses of chlorfenapyr and clothianidin insecticides were $50 \mu \mathrm{g} / \mathrm{bottle}$ and $150 \mathrm{\mu g} / \mathrm{bottle}$, respectively, for An. gambiae, Kisumu strain. When exposed to the diagnostic dose of each insecticide, Anopheles malaria vector populations in western Kenya were susceptible to both insecticides with 100\% mortality observed after $72 \mathrm{~h}$. Mortality of mosquitoes exposed to deltamethrin increased over time but did not reach 100\%. Mortality of Anopheles arabiensis from Nyando exposed to deltamethrin was $83 \%$ at 24 h, $88 \%$ at $48 \mathrm{~h}$ and $94.5 \%$ at $72 \mathrm{~h}$ while An. funestus from Ndhiwa was $89 \%$ at $24 \mathrm{~h}, 91.5 \%$ at $48 \mathrm{~h}$ and $94.5 \%$ at $72 \mathrm{~h}$.

Conclusion: Mosquitoes of western Kenya, despite being resistant to pyrethroids, are susceptible to chlorfenapyr and clothianidin. Field evaluations of the formulated product are needed.

Keywords: Anopheles, Anopheles gambiae sensu stricto, Anopheles arabiensis, Anopheles funestus, Chlorfenapyr, Clothianidin, Insecticide resistance

\footnotetext{
*Correspondence: s_agumba@yahoo.com

${ }^{1}$ Maseno University, Private Bag, Maseno, Kenya

Full list of author information is available at the end of the article
} 


\section{Background}

Considerable progress in reducing the global burden of malaria has been achieved since 2000. In Africa, prevalence estimated from national surveys declined by half from 2000 to 2015 while clinical cases declined by $40 \%$ [1]. More recently, gains in reducing malaria have slowed and in some countries, malaria burden has increased [2]. Sub-Saharan Africa shoulders the bulk of the malaria burden with $88 \%$ of malaria cases and $93 \%$ of malaria deaths occurring in this region [3]. There are likely multiple reasons for the slowed progress in reducing malaria, but one likely reason is the emergence and spread of insecticide resistance in the major malaria vectors, a development that may compromise chemical based malaria control interventions and thereby threaten malaria control and elimination efforts.

There are six classes of insecticides recommended for malaria vector control: pyrethroids, organochlorines, carbamates, organophosphates, pyrrole and neonicotinoids [4]. Pyrethroids are widely used for malaria vector control but resistance has developed in the major malaria vectors of Africa [5] and is now present in nearly every country in sub-Saharan Africa [6] (http://www.irmapper. $\mathrm{com} /$ ). Due to the threat posed by insecticide resistance, there has been an urgent call for alternative insecticides to supplement malaria vector control $[7,8]$. As new compounds are developed, it is essential to establish diagnostic concentrations to determine baseline susceptibility of malaria vectors and to enable surveillance of insecticide resistance once the insecticides are in use to guide National Malaria Control Programmes in the deployment and replacement of the insecticide-based vector control tools.

Chlorfenapyr is a slow acting toxin that acts by disrupting respiratory pathways and proton gradients through the uncoupling of oxidative phosphorylation in mitochondria [9]. It has a unique mode of action compared to insecticides currently used for public health; so far, shown there has been no evidence of cross resistance with insect neurotoxins that have widely been used for malaria vector control. Chlorfenapyr is an active ingredient (along with alpha-cypermethrin) on the Interceptor ${ }^{\circledR}$ G2 (BASF, Ludwigshafen Germany), a long-lasting insecticidal net that was recently recommended by the WHO Pesticide Evaluation Scheme [10]. In experimental hut trials, the Interceptor G2 caused higher mortality of wild mosquitoes compared to the WHOPES recommended standard Interceptor ${ }^{\circledR}$ net [11].

Clothianidin is a neonicotinoid insecticide which is chemically similar to nicotine. It acts on the central nervous system of insects as an agonist of acetylcholine and stimulates nicotine acetylcholine receptors (nAChR) [12] activating post-synaptic acetylcholine receptors but does not inhibit acetylene cholinesterase (ACh). High levels overstimulate and block the receptors, [13] causing paralysis and death [12]. Clothianidin is the active ingredient in SumiShield (developed by Sumitomo Chemical Company, Japan) and Fludora ${ }^{\circledR}$ Fusion (Bayer CropScience, Monheim, Germany) along with deltamethrin, an IRS formulation which was recently added to the WHO prequalification list of recommended insecticides [14]. This study estimated the diagnostic doses of chlorfenapyr and clothianidin insecticides in bottle bioassays using laboratory-reared Anopheles gambiae, Kisumu strain, and used the diagnostic dose identified to evaluate the susceptibility of wild Anopheles malaria vectors of western Kenya to chlorfenapyr, clothianidin and the pyrethroid deltamethrin.

\section{Methods}

Study sites

The study was conducted in three sub-Counties in western Kenya: Ndhiwa in Homa Bay County, Nyando in Kisumu County and Bumula in Bungoma County. The three sites experience perennial transmission of malaria with seasonal peaks between April to July and November to December coincident with the long and short rainy seasons respectively [15]. Malaria prevalence among children 6 months to 14 years as estimated in the most recent Malaria Indicator Survey was 38\% for the region despite high coverage with long-lasting insecticidal nets (LLINs) [16]. Most people live in traditional houses with mud walls plus thatched or corrugated iron roofs and practice agriculture as the major economic activity. The main crops grown in Ndhiwa sub-County are sugarcane and maize [17]. The primary malaria vector observed in this site is Anopheles funestus. Residents of Bumula sub-County grow cash crops such as sugar cane and tobacco and does horticulture farming. Anopheles gambiae sensu stricto (s.s.) is the primary vector in this area [18]. Nyando sub-county is a rice growing area providing larval habitats for Anopheles arabiensis throughout the year [19]. Pyrethroid resistance is widespread in western Kenya and has been observed in all three of the primary malaria vector species $[20,21]$.

\section{Mosquito sampling and processing}

Anopheles gambiae, Kisumu strain (pyrethroid susceptible) was reared at the Kenya Medical Research Institute's Center for Global Health Research (KEMRI-CGHR) insectary in a standard environment. Rearing methodology was based on Ochomo et al. [19] protocol. Wild adult Anopheles mosquitoes were sampled indoors using mouth aspirators while larvae were collected from small pools of water using standard dippers. Samples were transported to the insectary at KEMRI-CGHR and 
identified to species level using morphological keys [22]. Adult mosquitoes collected by indoor aspiration were held for $48 \mathrm{~h}$ post collection and then exposed to insecticides. Larvae were reared to adults and exposed when they were 3-5 days old. Members of the An. gambiae [23] and An. funestus complex [24] were identified by the polymerase chain reaction using primers specific for species known to occur in western Kenya. Mosquitoes from all sites were exposed to chlorfenapyr, clothianidin and deltamethrin insecticides for $1 \mathrm{~h}$. The diagnostic dose for deltamethrin was $12.5 \mu \mathrm{g} / \mathrm{ml}$, as recommended by WHO for monitoring resistance in the laboratory condition [25].

\section{Insecticides}

Technical-grade active ingredients of chlorfenapyr and clothianidin (Chem Service, Inc., West Chester, PA, USA) were used. Stock solutions were prepared for each insecticide by diluting the active ingredient in absolute ethanol, and storing in glass bottles, wrapped in aluminum foil, and at $4{ }^{\circ} \mathrm{C}$ while not being used. Working solutions were prepared from the stock solution. Field collected mosquitoes from two sites (Nyando and Ndhiwa) were exposed to a pyrethroid (deltamethrin) insecticide for comparison.

\section{Determination of the diagnostic doses of chlorfenapyr and clothianidin}

Mosquitoes were tested using the CDC bottle assay following Brodgon and Chan [26]. Given the slow rate of activity of these two insecticides [27, 28], mortality was recorded at 24-h intervals up to $72 \mathrm{~h}$. Ranges of insecticide concentrations were tested for chlorfenapyr $(0,10$, $20,30,40,50,60,70,80,90$, and $100 \mu \mathrm{g} / \mathrm{ml}$ ) and clothianidin $(0,50,100,150,200$ and $250 \mu \mathrm{g} / \mathrm{ml})$. Approximately $14 \mathrm{~h}$ after coating bottles with insecticide, 25 female adult An. gambiae, Kisumu (pyrethroid susceptible) (3-5 days old) were aspirated from the main colony and gently blown into each bottle. Mosquitoes were aspirated into the control bottle first, followed by the four insecticidecoated bottles. Once aspirated into each of the five bottles, the timer was started and time zero was recorded. The number of live and knocked down mosquitoes were recorded every $10 \mathrm{~min}$ for the $60 \mathrm{~min}$ exposure period. After $60 \mathrm{~min}$, the mosquitoes were gently aspirated from the bottle into clean paper cups, and provided with $10 \%$ sugar solution soaked in cotton wool during recovery period. All bottles were held vertically for the duration of the experiment. Dose response information of all tested concentration for both chlorfenapyr and clothianidin insecticides is found in Additional file 1.

\section{Statistical analysis}

Data from CDC bottle bioassays were subjected to log probit regression analysis [29] using SPSS statistics 20.0 $\mathrm{v}$ and LC50 and LC99 were calculated with 95\% confidence intervals. Efficacy of these compounds against wild Anopheles was calculated as percentage mortality, following World Health Organization (WHO) guidelines on insecticides susceptibility: mortality $\geq 98 \%$ indicates susceptibility, mortality less than $90 \%$ indicates the existence of resistance while if the observed mortality is between 90 and $97 \%$, the presence of resistant genes in the vector population must be confirmed [25].

\section{Results \\ Diagnostic dosage of chlorfenapyr and clothianidin insecticides using a susceptible strain}

Mortality of An. gambiae Kisumu strain exposed to clothianidin and chlorfenapyr is presented in Figs. 1 and 2 respectively. The $\mathrm{LC}_{50}$ and $\mathrm{LC}_{99}$ for both insecticides estimated by probit analysis are presented in Table 1. The LC50 for chlorfenapyr at $72 \mathrm{~h}$ was $55.4 \mu \mathrm{g} /$ bottle (p-value $<0.001$ ) while the LC50 for clothianidin was $143.5 \mu \mathrm{g} /$ bottle $(\mathrm{p}$-value $=0.04)$. For simplicity, the diagnostic doses selected for testing wild mosquitoes were $50 \mu \mathrm{g} /$ bottle for chlorfenapyr and $150 \mu \mathrm{g} /$ bottle for clothianidin. Chlorfenapyr had no knockdown effect upon the susceptible $A n$. gambiae Kisumu strain after exposure while clothianidin had a mean knockdown of $11.2 \%$ at $60 \mathrm{~min}$.

\section{Efficacy of chlorfenapyr, clothianidin and deltamethrin against malaria vector populations of western Kenya} A total of 2379 mosquitoes were tested for susceptibility to chlorfenapyr, clothianidin and deltamethrin by PCR as shown in Fig. 3, all mosquitoes tested from Nyando subcounty were An. arabiensis (1133/1133), 96.5\% (386/400) of mosquitoes tested from Bumula sub-County were An. gambiae s.s. and $3.5 \%(14 / 400)$ were An. arabiensis while $97.3 \%$ (827/850) of mosquitoes tested from Ndhiwa sub-county were An. funestus and 2.7\% (23/400) were An. arabiensis. Anopheles populations of western Kenya showed $100 \%$ mortality in CDC-Bottle bioassays at $72 \mathrm{~h}$ post-exposure to both chlorfenapyr and clothianidin with resistance observed to deltamethrin insecticide in two study sites, even at $72 \mathrm{~h}$ post-exposure. Mortality $72 \mathrm{~h}$ after deltamethrin exposure was $90.0 \%$ among $A n$. arabiensis in Nyando sub-county and $94.5 \%$ among $A n$. funestus in Ndhiwa sub-County. Chlorfenapyr had no knockdown effect on wild mosquitoes with $0 \%$ knockdown at $60 \mathrm{~min}$ in all three populations tested. The mean knockdown rates at $60 \mathrm{~min}$ of wild $A n$. arabiensis, $A n$. funestus and An. gambiae s.s. exposed to clothianidin 


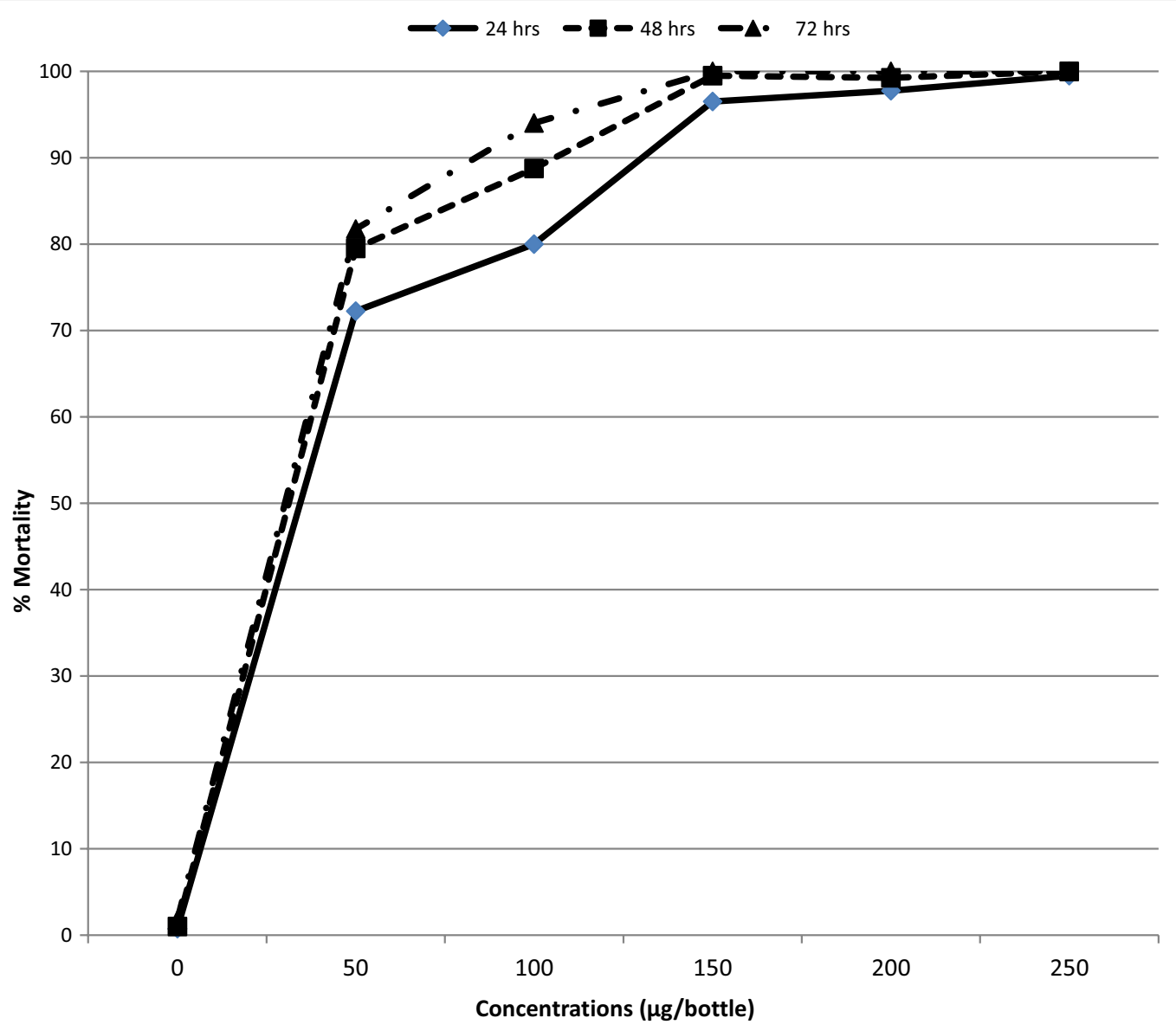

Fig. 1 Mortality \% of An. gambiae s.s (Kisumu strain) to varying concentrations of clothianidin insecticides

were $12.5 \%, 4.3 \%$ and $0.5 \%$ respectively. Knockdown at 60 min after exposure to deltamethrin was $90.5 \%$ among An. arabiensis from Nyando and $86 \%$ among An. funestus from Ndhiwa. Table 2 shows results for different holding times by sub-County, which reflect different species compositions.

\section{Discussion}

As new active ingredients are developed, it is essential to establish diagnostic concentrations to determine baseline susceptibility of malaria vectors and to enable monitoring of insecticide resistance once the insecticides are in use to guide National Malaria Control Programmes in the deployment, rotation and replacement of the insecticide based vector control tools. This study determined the diagnostic doses of chlorfenapyr (a pyrrole) and clothianidin (a neonicotinoid) insecticides in CDC bottle bioassays using laboratory-reared An. gambiae, Kisumu strain. This study used the diagnostic dose to evaluate the susceptibility of three wild Anopheles malaria vectors of western Kenya. The diagnostic doses for chlorfenapyr and clothianidin were defined as the lowest concentration that achieves $100 \%$ mortality within $72 \mathrm{~h}$ after a 60 min exposure. Based on this criterion, the diagnostic doses were determined to be $50 \mu \mathrm{g} /$ bottle for chlorfenapyr and $150 \mu \mathrm{g} / \mathrm{ml}$ for clothianidin. These diagnostic doses are important in providing baseline data for monitoring insecticides resistance when deployed for malaria vector control in western Kenya. Three field populations of primarily An. gambiae, An. arabiensis and An. funestus, respectively, were subsequently exposed to these diagnostic doses resulting in $100 \%$ mortality at $72 \mathrm{~h}$ for both insecticides in all three species tested, confirming baseline susceptibility.

The knockdown rate for both the laboratory strain of An. gambiae, Kisumu strain and field collected mosquitoes at $60 \mathrm{~min}$ after exposure was low for chlorfenapyr and clothianidin, despite $100 \%$ mortality at $72 \mathrm{~h}$. In comparison, pyrethroid insecticides are known for their strong knockdown effects. Even among field populations of An. arabiensis and An. funestus with some resistance to pyrethroids, exposure to deltamethrin resulted in $90.5 \%$ 


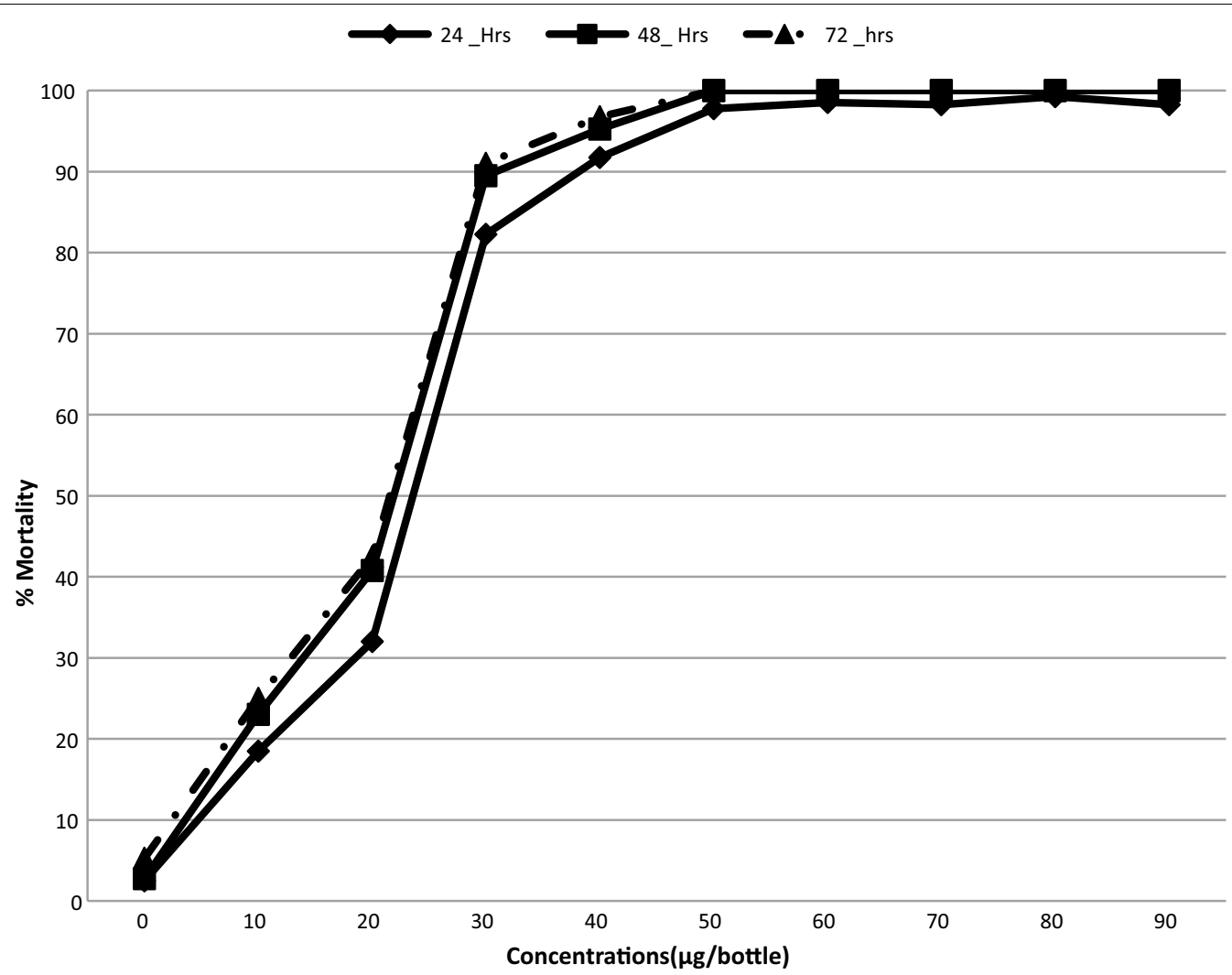

Fig. 2 Mortality percentage of An. gambiae s.s (Kisumu strain) to varying concentrations of chlorfenapyr

Table 1 Probit analysis results of An. gambiae s.s. mortality $72 \mathrm{~h}$ after exposure to chlorfenapyr and clothianidin

\begin{tabular}{lll}
\hline & Chlorfenapyr & Clothianidin \\
\hline Number exposed & 400 & 400 \\
$\mathrm{LD}_{50 \%}\left(95 \% \mathrm{Cl}^{\mathrm{a}}\right)$ & $16.8(13.6-19.9)$ & $27.576(5.8-41.2)$ \\
$\mathrm{LD}_{99 \%}\left(95 \% \mathrm{Cl}^{\mathrm{a}}\right)$ & $55.4(42.8-85.7)$ & $143.5(100.2-503.9)$ \\
Diagnostic dose & $50 \mu \mathrm{g} / \mathrm{ml}$ & $150 \mu \mathrm{g} / \mathrm{ml}$ \\
Chi square $\left(\mathrm{X}^{2}\right)$ & 117.5 & 13.4 \\
p-value & 0.001 & 0.040
\end{tabular}

${ }^{a}$ Upper and lower limits with $95 \%$ confidence intervals $x^{2}$ (Chi square)

and $86 \%$ knockdown, respectively, at $60 \mathrm{~min}$. While the rapid knockdown and killing effect is often considered an important component of the efficacy of pyrethroids, modelling studies have suggested that this mode of action is more likely to select for insecticide resistance [30]. In contrast, slow acting insecticides such as clothianidin and chlorfenapyr could impose less selection pressure for resistance as mosquitoes may survive long enough to lay eggs. Despite the slow acting effect of chlorfenapyr and clothianidin, these insecticides may be able to reduce malaria transmission as mortality was $100 \%$ at $72 \mathrm{~h}$ in all populations tested and the external incubation period of Plasmodium falciparum is a minimum of 8 days [31]. For deltamethrin, mortality did not substantially increase over $72 \mathrm{~h}$ suggesting that mosquitoes that survive initial exposure to pyrethroids may be able to sustain transmission of malaria [32].

Resistance to pyrethroid insecticides in western Kenya was first reported in An. gambiae s.s. following the implementation of small-scale trials of permethrin treated nets [33]. Initial studies indicated that elevated oxidase and esterase enzymes were associated with increased permethrin tolerance [34]. Subsequently, a target site mutation $(k d r$ L1014S) was described in western Kenya and was associated with pyrethroid resistance [8]. Following the scale-up of insecticide treated nets in Kenya, the frequency of the L1014S mutation increased from $<5 \%$ to near fixation in $A n$. gambiae s.s. [15]. Resistance to pyrethroids was subsequently reported in An. arabiensis and An. funestus [19, 35]. The resistance mechanisms in An. arabiensis and An. funestus are not well described, but are likely metabolic detoxification enzymes as the $k d r$ mutations in 


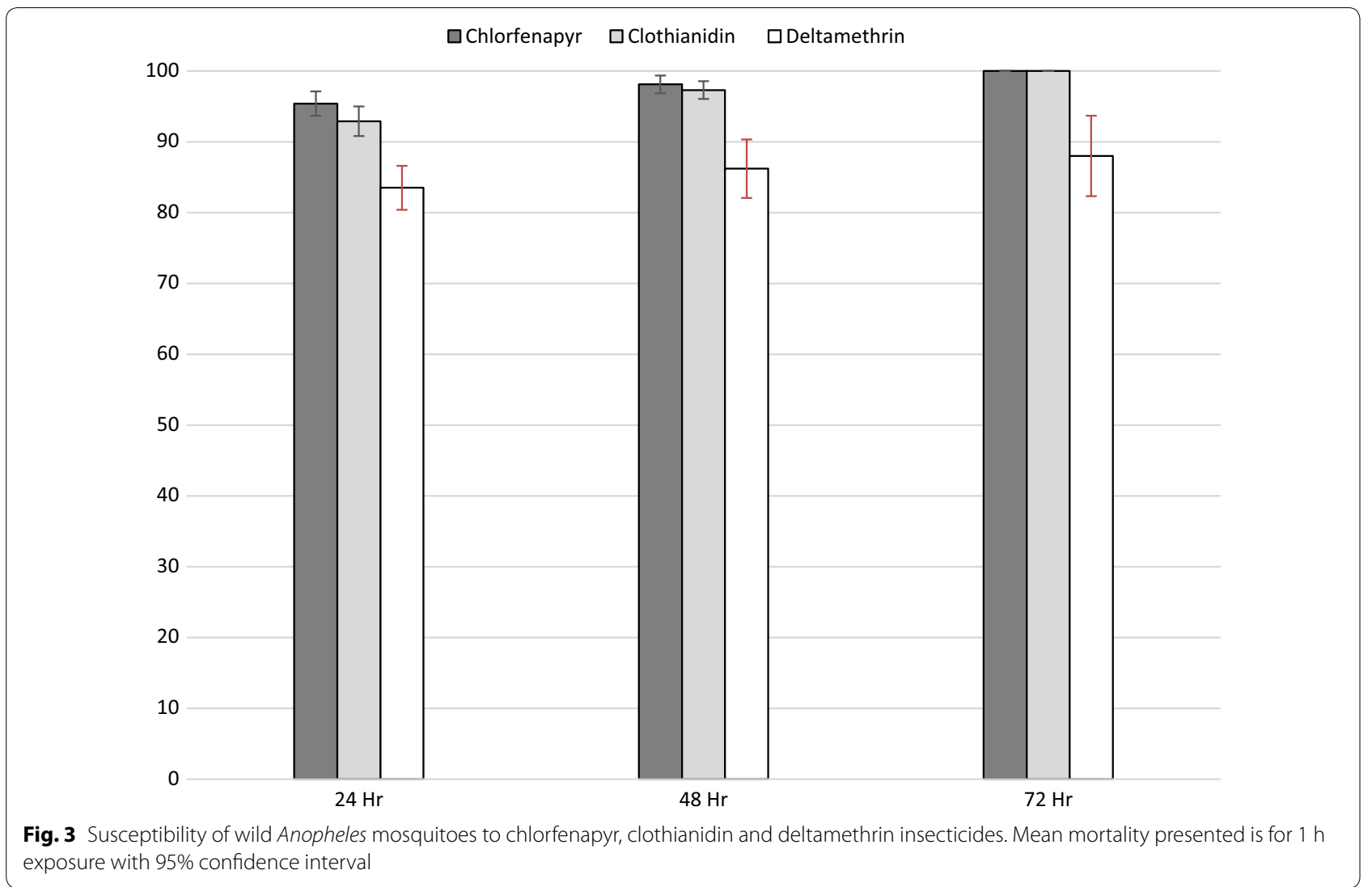

Table 2 Percentage mortality of wild mosquito populations in response to chlorfenapyr, clothianidin and deltamethrin diagnostic doses

\begin{tabular}{|c|c|c|c|c|c|c|}
\hline \multirow[t]{2}{*}{ Site/population } & \multirow[t]{2}{*}{ Insecticide } & \multirow[t]{2}{*}{ Sample size (N) } & \multirow{2}{*}{$\begin{array}{l}\% \text { Knockdown } \\
\text { at } 60 \mathrm{~min}\end{array}$} & \multicolumn{3}{|c|}{$\%$ Mortality } \\
\hline & & & & $24 \mathrm{~h}$ & $48 \mathrm{~h}$ & $72 \mathrm{~h}$ \\
\hline \multirow{3}{*}{$\begin{array}{l}\text { Nyando } \\
\text { An. arabiensis }\end{array}$} & Chlorfenapyr & 407 & 0 & 94.6 & 96.1 & 100.0 \\
\hline & Clothianidin & 415 & 12.5 & 92.8 & 99.3 & 100.0 \\
\hline & Deltamethrin & 200 & 90.5 & 83.0 & 88.0 & 90.0 \\
\hline \multirow{3}{*}{$\begin{array}{l}\text { Ndhiwa } \\
\text { An. funestus }\end{array}$} & Chlorfenapyr & 343 & 0 & 98.0 & 99.4 & 100 \\
\hline & Clothianidin & 303 & 4.3 & 89.0 & 97.0 & 100 \\
\hline & Deltamethrin & 200 & 86.0 & 89.1 & 91.5 & 94.5 \\
\hline \multirow{3}{*}{$\begin{array}{l}\text { Bumula } \\
\text { An. gambiae s.s. }\end{array}$} & Chlorfenapyr & 200 & 0 & 92.7 & 98.0 & 100 \\
\hline & Clothianidin & 200 & 0.5 & 98.1 & 99.5 & 100 \\
\hline & Deltamethrin & ND & ND & ND & ND & ND \\
\hline
\end{tabular}

An. arabiensis are rare in western Kenya [36] and have never been reported from An. funestus. The high levels of susceptibility of field populations to chlorfenapyr and clothianidin indicate a lack of cross-resistance with pyrethroid insecticides. The target sites of both insecticides are different from that of pyrethroids and the lack of cross-resistance indicates that enzymes involved in the metabolic detoxification of pyrethroids do not affect either chlorfenapyr or clothianidin. Furthermore, chlorfenapyr is considered a pro-insecticide that is activated by oxidase enzymes suggesting a potential for negative cross-resistance [37]. Resistance to chlorfenapyr and clothianidin have not yet been described in mosquitoes; but additional information on the molecular mechanisms of insecticide resistance may help to predict the potential for cross-resistance and to guide 
National Malaria Control Programmes in the selection of insecticides for ITNs and IRS.

The Global Plan for Insecticide Resistance Management (GPIRM) has four main strategies (rotation of insecticides, combination of interventions, mosaic spraying and mixtures) for vector control. Until 2017, only four classes of insecticides were recommended for use in public health-pyrethroids, organochlorines, organophosphates and carbamates; resistance has been reported for each class [14]. There are now two additional classes of insecticides available for use in public health and there are already products formulated with these insecticides. The Interceptor G2 (BASF, Ludwigshafen Germany) is an LLIN treated with alpha cypermethrin plus chlorfenapyr. Experimental hut studies indicate significantly higher mosquito mortality due to the Interceptor G2 compared to pyrethroid only nets, even after washing [11]. SumiShield (Sumitomo Chemical, Japan) and Fludora ${ }^{\circledR}$ Fusion (Bayer Environmental Science, Germany) are IRS products with clothianidin as the active ingredient. SumiShield was demonstrated to last up to 6 months in various surfaces in experimental huts [38]. With the availability of an LLIN treated with a pyrethroid plus chlorfenapyr and two IRS products with clothianidin along with LLINs treated with a synergist (piperonyl butoxide), management of insecticide resistance among wild Anopheles populations is more feasible. However, despite general recommendations from the GPIRM, specific guidance is lacking on how to optimally deploy these tools.

The CDC bottle bioassay used for this study offers important advantages. The bottle bioassay allows for substantial flexibility, particularly for assessing resistance to new insecticides, which is useful since diagnostic doses and papers for the WHO tub assay are not yet available. Nevertheless, the approaches used in this study also have limitations. Clothianidin has poor solubility in most solvents used in bottle assays. Clothianidin has very low solubility in acetone and preliminary studies using this solvent have given widely inconsistent results, presumably due to an uneven coating of the bottle. For this reason, the study choose to use ethanol. Solubility of clothianidin is also low in ethanol but this was resolved by allowing the mixtures of clothianidin and ethanol to sit for up to 3 days and confirming the absence of crystallization before coating bottles. For both insecticides, the slow acting effects meant that mosquitoes exposed in the bioassays had to be held for up to 3 days. This presents challenges as mosquitoes exposed in control tubes are more likely to die with increasing holding periods, which may invalidate the bioassays. Careful rearing procedures are required to ensure high quality bioassays with these slower acting insecticides.

\section{Conclusion}

In summary, the estimated diagnostic doses for chlorfenapyr and clothianidin in CDC bottle bioassays as $50 \mathrm{ml} /$ bottle and $150 \mathrm{ml} /$ bottle respectively. Subsequently, the study demonstrated full susceptibility to these insecticides in wild populations of An. gambiae, $A n$. arabiensis, and An. funestus that are resistant to pyrethroids. These results indicate that chlorfenapyr and clothianidin may be effective against the three main malaria vectors in western Kenya and they serve as a baseline for monitoring resistance should the Kenya National Malaria Control Programme implement LLINs or IRS using these insecticides.

\section{Additional file}

Additional file 1. Table S1. Detailed results of the CDC bottle assay for different concentrations during determination of diagnostic concentrations for chlorfenapyr insecticide. Table $\mathbf{S 2}$. Detailed results of the CDC bottle assay performed for different concentrations during determination of diagnostic concentrations for clothianidin insecticides. Table S3. Control results for both chlorfenapyr and clothianidin insecticides respectively.

\section{Abbreviations}

CDC: Centre for Disease Control and Prevention; CGHR: Centre Global Health Research; GPIRM: Global Plan for Insecticide Resistance Management; LD: lethal dose; KEMRI: Kenya Medical Research Institute; WHO: World Health Organization; WHOPES: World Health Organization Pest Evaluation Scheme.

\section{Acknowledgements}

We are grateful to the KEMRI-CGHR entomology laboratory team especially Richard Amito and Duncan Omondi for their effort in samples collection as well as molecular team under the guidance of Celestine Wekesa and Mathew Kipsum. Last but not least, this research might have not been completed without the support grant from the National Commission on Science, Technology and Innovation (NACOSTI). We thank the Director KEMRI for the permission to publish this data.

\section{Disclaimer}

The findings and conclusions in this report do not necessarily reflect the official position of the U.S. Department of Health and Human Services or the U.S. Centers for Disease Control and Prevention. Use of trade names is for identification only and does not imply endorsement by the U.S. Department of Health and Human Services or the U.S. Centers for Disease Control and Prevention.

\section{Authors' contributions}

$S A, E O, J G$ designed the study. The sample collections were conducted by SA. SO and SA contributed to bioassays. SA, EO, JK, SM, LO and BG contributed to writing the draft. Species identification was done by SA. All authors read and approved the final manuscript.

\section{Funding}

This study was funded by National Research Fund 1st call of abstract 2016/2017 grant from the National Commission on Science, Technology and Innovation (NACOSTI) Kenya.

\section{Availability of data}

The dataset supporting the conclusion is available from the corresponding author upon request. 


\section{Ethics approval and consent to participate}

KEMRI-CGHR Ethical review committee. KEMRI SERU SSC 2776 and the consent to The protocol was reviewed and approved by the Scientific Ethics Review Unit of the Kenya Medical Research Institute (SSC 2776) and by the Institutional Review Board of the US Centers for Disease Control and Prevention (IRB 6728) through a reliance agreement with KEMRI.

\section{Consent for publication}

Not applicable.

\section{Competing interests}

The authors declared that they have no competing interests.

\section{Author details}

${ }_{1}^{1}$ Maseno University, Private Bag, Maseno, Kenya. ${ }^{2}$ Centre for Global Health Research, Kenya Medical Research Institute, PO Box 1578-40100, Kisumu, Kenya. ${ }^{3}$ Division of Parasitic Diseases and Malaria, Center for Global Health, Centers for Disease Control and Prevention, Atlanta, GA 30333, USA.

Received: 6 December 2018 Accepted: 1 July 2019

Published online: 17 July 2019

\section{References}

1. Bhatt S, Weiss DJ, Cameron E, Bisanzio D, Mappin B, Dalrymple U, et al. The effect of malaria control on Plasmodium falciparum in Africa between 2000 and 2015. Nature. 2015;526:207-11.

2. WHO. World malaria report, 2017. Geneva: World Health Organization; 2017. https://www.who.int/malaria/publications/world-malaria-repor t-2017/en/. Accessed Dec 2017.

3. WHO. World malaria report 2018. Geneva: World Health Organization; 2018. https://www.who.int/malaria/publications/world-malaria-repor t-2018/report/en/. Accessed Dec 2018.

4. WHO. Recommended insecticides for indoor residual spraying against malaria vectors. Geneva: World Health Organization; 2018. https://www. who.int/neglected_diseases/vector_ecology/vector-control/Insecticid es_IRS_22_September_2018.pdf. Accessed Jan 2018.

5. Kleinschmidt I, Bradley J, Knox TB, Mnzava AP, Kafy HT, Mbogo C, et al. Implications of insecticide resistance for malaria vector control with longlasting insecticidal nets: a WHO-coordinated, prospective, international, observational cohort study. Lancet Infect Dis. 2018;18:640-9.

6. Knox TB, Juma EO, Ochomo EO, Jamet HP, Ndungo L, Chege P, et al. An online tool for mapping insecticide resistance in major Anopheles vectors of human malaria parasites and review of resistance status for the Afrotropical region. Parasit Vectors. 2014;7:76

7. Zaim M, Guillet P. Alternative insecticides: an urgent need. Trends Parasitol. 2002;18:161-3.

8. Ranson H, N'guessan R, Lines J, Moiroux N, Nkuni Z, Corbel V. Pyrethroid resistance in African anopheline mosquitoes: what are the implications for malaria control? Trends Parasitol. 2011;27:91-8.

9. Black BC, Hollingworth RM, Ahammadsahib Kl, Kukel CD, Donovan $\mathrm{S}$. Insecticidal action and mitochondrial uncoupling activity of AC-303,630 and related halogenated pyrroles. Pestic Biochem Physiol. 1994:50:115-28.

10. Camara S, Ahoua Alou LP, Koffi AA, Clegban YCM, Kabran JP, Koffi FM, et al. Efficacy of Interceptor ${ }^{\circledR}$ G2, a new long-lasting insecticidal net against wild pyrethroid-resistant Anopheles gambiae s.s. from Côte d'Ivoire: a semi-field trial. Parasite. 2018:25:42.

11. Bayili K, N'do S, Namountougou M, Sanou R, Ouattara A, Dabiré RK, et al. Evaluation of efficacy of Interceptor ${ }^{\circledR} \mathrm{G} 2$, a long-lasting insecticide net coated with a mixture of chlorfenapyr and alpha-cypermethrin, against pyrethroid resistant Anopheles gambiae s.I. in Burkina Faso. Malar J. 2017;16:190.

12. Krupke $\mathrm{CH}$, Long EY. Intersections between neonicotinoid seed treatments and honey bees. Curr Opin Insect Sci. 2015;10:8-13.

13. Yamamoto I. Nicotine to nicotinoids: 1962 to 1997. In: Yamamoto I, Casida JE, editors. Nicotinoid insecticides and the nicotinic acetylcholine receptor. Berlin: Springer; 1999. p. 3-27.

14. Prequalification Vector Control. https://www.who.int/pq-vector-control/ prequalified-lists/en/. Accessed Jan 2019.
15. Mathias DK, Ochomo E, Atieli F, Ombok M, Bayoh MN, Olang G, et al. Spatial and temporal variation in the kdr allele L1014S in Anopheles gambiae s.s. and phenotypic variability in susceptibility to insecticides in Western Kenya. Malar J. 2011;10:10

16. Malaria Operation Plan, Kenya, FY 2017. In: Presidential's Malaria Initiative. https://www.pmi.gov/docs/default-source/default-document-library/ malaria-operational-plans/fy17/fy-2017-kenya-malaria-operational-plan. pdf?sfvrsn=6. Accessed Oct 2017

17. Ndhiwa is a settlement in Kenya's Nyanza Province. https://www.ngcdf go.ke/index.php/2015-07-28-04-03-42/constituency/250-ndhiwa. Accessed Mar 2019

18. Bayoh MN, Mathias DK, Odiere MR, Mutuku FM, Kamau L, Gimnig JE, et al. Anopheles gambiae: historical population decline associated with regional distribution of insecticide-treated bed nets in western Nyanza Province, Kenya. Malar J. 2010;9:62.

19. Ochomo E, Bayoh MN, Brogdon WG, Gimnig JE, Ouma C, Vulule JM, et al. Pyrethroid resistance in Anopheles gambiae s.s. and Anopheles arabiensis in western Kenya: phenotypic, metabolic and target site characterizations of three populations. Med Vet Entomol. 2013;27:156-64.

20. Omondi S, Mukabana WR, Ochomo E, Muchoki M, Kemei B, Mbogo C, et al. Quantifying the intensity of permethrin insecticide resistance in Anopheles mosquitoes in western Kenya. Parasit Vectors. 2017;10:548.

21. Kawada H, Dida GO, Ohashi K, Komagata O, Kasai S, Tomita T, et al. Multimodal pyrethroid resistance in malaria vectors, Anopheles gambiae s.s. Anopheles arabiensis, and Anopheles funestus s.s. in western Kenya. PLoS ONE. 2011;6:e22574.

22. Gillies MT, De Meillon B. The Anophelinae of Africa south of the Sahara (Ethiopian zoogeographical region). 1968.

23. Collins FH, Mendez MA, Rasmussen MO, Mehaffey PC, Besansky NJ, Finnerty V. A ribosomal RNA gene probe differentiates member species of the Anopheles gambiae complex. Am J Trop Med Hyg. 1987:37:37-41.

24. Wilkins EE, Howell PI, Benedict MQ. IMP PCR primers detect single nucleotide polymorphisms for Anopheles gambiae species identification, Mopti and Savanna rDNA types, and resistance to dieldrin in Anopheles arabiensis. Malar J. 2006;5:125.

25. WHO. Test procedures for insecticide resistance monitoring in malaria vector mosquitoes. 2nd edition. https://www.who.int/malaria/publicatio ns/atoz/9789241511575/en/. Accessed Oct 2018.

26. Brogdon W, Chan A. Guideline for evaluating insecticide resistance in vectors using the CDC bottle bioassay. USA: CDC Atlanta; 2010

27. N'Guessan R, Boko P, Odjo A, Knols B, Akogbeto M, Rowland M. Control of pyrethroid-resistant Anopheles gambiae and Culex quinquefasciatus mosquitoes with chlorfenapyr in Benin. Trop Med Int Health. 2009;14:389-95.

28. Tan J, Galligan JJ, Hollingworth RM. Agonist actions of neonicotinoids on nicotinic acetylcholine receptors expressed by cockroach neurons. Neurotoxicology. 2007;28:829-42.

29. Finney DJ. Probit analysis. 3rd ed. New York: Cambridge University Press; 1971.

30. Read AF, Lynch PA, Thomas MB. How to make evolution-proof insecticides for malaria control. PLoS Biol. 2009;7:e1000058.

31. Killeen GF, McKenzie FE, Foy BD, Schieffelin C, Billingsley PF, Beier JC. A simplified model for predicting malaria entomologic inoculation rates based on entomologic and parasitologic parameters relevant to control. Am J Trop Med Hyg. 2000;62:535-44.

32. Ochomo EO, Bayoh NM, Walker ED, Abongo BO, Ombok MO, Ouma C, et al. The efficacy of long-lasting nets with declining physical integrity may be compromised in areas with high levels of pyrethroid resistance. Malar J. 2013;12:368.

33. Vulule JM, Beach RF, Atieli FK, Roberts JM, Mount DL, Mwangi RW. Reduced susceptibility of Anopheles gambiae to permethrin associated with the use of permethrin-impregnated bednets and curtains in Kenya. Med Vet Entomol. 1994:8:71-5.

34. Vulule JM, Beach RF, Atieli FK, MCAllister JC, Brogdon WG, Roberts JM, et al. Elevated oxidase and esterase levels associated with permethrin tolerance in Anopheles gambiae from Kenyan villages using permethrinimpregnated nets. Med Vet Entomol. 1999;13:239-44.

35. Kawada H, Ohashi K, Dida GO, Sonye G, Njenga SM, Mwandawiro C, et al. Insecticidal and repellent activities of pyrethroids to the three major pyrethroid-resistant malaria vectors in western Kenya. Parasit Vectors. $2014 ; 7: 208$ 
36. Ochomo E, Subramaniam K, Kemei B, Rippon E, Bayoh NM, Kamau L, et al. Presence of the knockdown resistance mutation, Vgsc-1014F in Anopheles gambiae and An. arabiensis in western Kenya. Parasit Vectors. 2015;8:616.

37. Raghavendra K, Barik TK, Sharma P, Bhatt RM, Srivastava HC, Sreehari U, et al. Chlorfenapyr: a new insecticide with novel mode of action can control pyrethroid resistant malaria vectors. Malar J. 2011;10:16.

38. Ngwej LM, Hattingh I, Mlambo G, Mashat EM, Kashala JCK, Malonga FK, et al. Indoor residual spray bio-efficacy and residual activity of a clothianidin-based formulation (SumiShield ${ }^{\circledR}$ 50WG) provides long persistence on various wall surfaces for malaria control in the Democratic Republic of the Congo. Malar J. 2019;18:72.

\section{Publisher's Note}

Springer Nature remains neutral with regard to jurisdictional claims in published maps and institutional affiliations.
Ready to submit your research? Choose BMC and benefit from:

- fast, convenient online submission

- thorough peer review by experienced researchers in your field

- rapid publication on acceptance

- support for research data, including large and complex data types

- gold Open Access which fosters wider collaboration and increased citations

- maximum visibility for your research: over $100 \mathrm{M}$ website views per year

At BMC, research is always in progress.

Learn more biomedcentral.com/submissions 